\title{
The Robust Derivative Code for Object Recognition
}

\author{
Hainan Wang ${ }^{1,4}$, Baochang Zhang ${ }^{1}$, Hong Zheng ${ }^{1}$, Yao Cao ${ }^{1}$, Zhenhua Guo ${ }^{2}$, \\ Chengshan Qian ${ }^{3}$ \\ ${ }^{1}$ Science and Technology on Aircraft Control Laboratory, Beihang University, Beijing, China \\ [bczhang@buaa.edu.cn; whnhnhn@163.com; julyana@buaa.edu.cn;] \\ ${ }^{2}$ Graduate school of Shenzhen, Shenzhen, Tsinghua University \\ [zhenhua.guo@sz.tsinghua.edu.cn] \\ ${ }^{3}$ School of Computer \& Software, Nanjing University of Information Science \& Technology, Nanjing, China \\ [qianchengshan@nuist.edu.cn] \\ 4 School of Mechanical Engineering, Guizhou University, Guiyang, China \\ *Corresponding author: Baochang Zhang
}

Received August 10, 2016; revised October 23, 2016; accepted November 13, 2016; published January 31, 2017

\begin{abstract}
This paper proposes new methods, named Derivative Code (DerivativeCode) and Derivative Code Pattern (DCP), for object recognition. The discriminative derivative code is used to capture the local relationship in the input image by concatenating binary results of the mathematical derivative value. Gabor based DerivativeCode is directly used to solve the palmprint recognition problem, which achieves a much better performance than the state-of-art results on the PolyU palmprint database. A new local pattern method, named Derivative Code Pattern (DCP), is further introduced to calculate the local pattern feature based on Dervativecode for object recognition. Similar to local binary pattern (LBP), DCP can be further combined with Gabor features and modeled by spatial histogram. To evaluate the performance of DCP and Gabor-DCP, we test them on the FERET and PolyU infrared face databases, and experimental results show that the proposed method achieves a better result than LBP and some state-of-the-arts.
\end{abstract}

Keywords: Object Recognition, Derivative Code, Local Pattern, Gabor wavelet

A preliminary version of this paper appeared in IEEE ICIA 2012, Shenzhen China. This version includes a concrete analysis and supporting implementation results on new datasets. This work was supported in part by the Natural Science Foundation of China under Contract 61272052 and 61473086, in part by PAPD and CICAEET. The work of B. Zhang was supported by the Program for New Century Excellent Talents University within the Ministry of Education, China, and Beijing Municipal Science \& Technology Commission Z161100001616005. Baochang zhang and Hainan Wang had the same contribution to the paper. 


\section{Introduction}

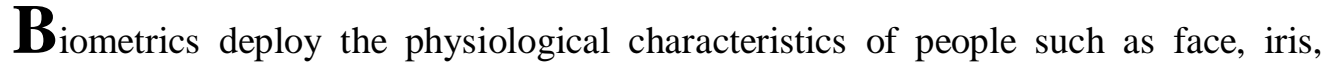
palmprint, gait, for identity recognition, which provide more stable ways than other traditional methods such as PIN, Key, ID cards [1,2,3,4]. With the development of the information technology, Biometrics technologies had changed human life, i.e., face recognition is widely used in the video surveillance. Faces and palmprint images contain much detailed information to discriminate between different people [5,6, 7]. Especially, the rich intensity information in the texture images varies because it suffers from changes of illumination settings, viewpoints, and occlusions[8,9,37-39].

Many statistic methods such as principal component analysis (PCA) [19, 20], linear discriminant analysis (LDA) [21, 22], kernel principal component Analysis (KPCA) [23] and kernel fisher discriminant analysis (KFDA) [24] have been widely investigated due to high efficiency to capture variance information among the training set. The PCA-based techniques exploit the low-dimensional representation of the objects, while discriminative LDA-based algorithms deal directly with the discrimination between classes. In the local kernel analysis (LFA) technique, a dense set of local-topological fields are developed to extract local features. To get more detailed description for faces or palmprint, steerable filters $[1,4]$ or Gabor filters $[35,36]$ are proposed to extract the scale and orientation information contained in the input images. However, the filter's response of the input image is not very desirable as noise or variation exists in the input object. Many other approaches are proposed to compute the more stable features, such as sparse representation [5, 6,11,17, 31-34], which employed sparse coding of original features. As another effective way, the ordinal method can calculate the sign of the response value. For example, local binary pattern (LBP) and its variants have become those of the most popular ways toward high performance by modeling the distribution of the local pattern features [7]. LBP is calculated based on the derivative binary pattern feature, which calculate the relationship between one point and its neighbors. The performance of the LBP histogram on texture understanding and object recognition shows that the derivative information can be a powerful way for object representation. In this paper, we investigate the derivative binary code for object recognition, based on which a new local pattern, named derivative code pattern (DCP), is further introduced to calculate the local pattern information for face recognition.

To evaluate the proposed methods, we focus on face and palmprint recognition problems. In the palmprint identification, Gabor ordinal feature [8] is the state-of-the-art method, which is used to validate our method, as the proposed method can also be considered as new ordinal feature. In face recognition, LBP has been widely used in the field of face recognition $[7,9,10]$, which achieves much better performance than other traditional approaches, such as Eigenface, Fisherface, and so on. To further validate the performance of the proposed method, sparse representation classifier and its variants are also included in our experiments [5, 31-34]. In the experimental part, the PolyU palmprint database, Polyu 
infrared database and FERET are used to compare the proposed methods with related methods.

The rest of the paper is organized as follows. Section 2 introduces Derivative code and its local pattern feature for object representation. In section 3, comparative experiments on three public databases are conducted to evaluate the performance of the proposed methods and some state-of-the-art results. Finally, conclusions are drawn in section 4 . The paper is an extension of our conference paper [26].

\section{Derivative Code and Its Local Pattern for object recognition}

In this section, we first briefly describe LBP, and then introduce the proposed robust derivative code for object recognition.

\subsection{The Robust Derivative Code descriptor}

To extract the robust representation from local neighborhood in the image, LBP is defined as a gray-scale invariant texture measure tool to model texture images, which later has shown excellent performance in many comparative studies. LBP is very excellent in terms of speed and discrimination performance $[9,10]$. The LBP operator labels the pixels of an image by thresholding the $3 \times 3$ neighborhood of each pixel with the value of the central pixel and concatenating the results to form a number. A LBP is represented as the concatenation of the binary gradient directions, which is called a micro-pattern. The histograms of these micro-patterns contain information of the distribution of the edges, spots, and other local features in an image. LBP has been successfully way to model faces. Different from traditional statistic learning methods, i.e., PCA and LDA, which need to tune a large number of parameters, LBP is very efficient due to its easy-to-compute feature extraction operation and simple matching strategy. The nature behind LBP is actually derivative operation $[9,10]$, which is also the basic idea of local derivative pattern (LDP) [9]. In a summary, LBP actually encodes the binary results of first-order derivative among local neighbours by using a simple threshold function as shown in [10], which is incapable of describing more detailed information. Differently the $n^{\text {th }}$-order LDP is defined to encode the $(n-1)^{\text {th }}$-order derivative direction variances based on a binary coding function. In this scheme, LBP is conceptually regarded as the non-directional first-order LDP, because LBP encodes 8-direction first-order derivative binary result.

\subsubsection{Derivative Code}

In this section, we describe the proposed derivative code and its pattern for object representation. Derivative code is proposed to encode the neighborhood derivative binary results in a local region. Given an image $I$, and let $I(x, y)$ be a point in $I$, the derivative feature of point $I(x, y)$ along $0^{\circ}, 45^{\circ}, 90^{\circ}$ and $135^{\circ}$ directions are denoted as $I_{\alpha, d}^{\prime}(x, y)$ with $\alpha=\left[0^{\circ}, 45^{\circ}, 90^{\circ}, 135^{\circ}\right]$. Here $d$ is defined as the distance between the given point and the neighboring points in four directions (as shown in Fig. 1, where $d=1$ ). The four derivatives 
at $I(x, y)$ can be written as:

$$
\begin{gathered}
I_{0^{\circ}, d}^{\prime}(x, y)=I(x, y)-I(x-d, y), \\
I_{45^{\circ}, d}^{\prime}(x, y)=I(x, y)-I(x-d, y-d), \\
I_{90^{\circ}, d}^{\prime}(x, y)=I(x, y)-I(x, y-d), \\
I_{135^{\circ}, d}^{\prime}(x, y)=I(x, y)-I(x+d, y-d) .
\end{gathered}
$$

The threshold functions $f\left(I^{\prime}{ }_{\alpha, d}(x, y)\right)$ is used to encode the binary results for each derivative direction. And then the so-called DerivativeCode, in given direction $\alpha$, is defined as:

$$
f\left(I_{\alpha, d}^{\prime}(x, y)\right)=\left\{\begin{array}{l}
1, \text { if } \quad I_{\alpha, d}^{\prime}(x, y) \geq 0 \\
0, \text { if } \quad I_{\alpha, d}^{\prime}(x, y)<0
\end{array}\right.
$$

\subsubsection{Derivative Code Pattern (DCP)}

The DerivativeCode is the pixel-based method to extract the directional feature, and DCP derived from DerivativeCode in the direction $\alpha$ is accordingly defined as:

$$
\begin{aligned}
D C P^{\prime}{ }_{\alpha, d}(x, y)=\{ & f\left(I_{\alpha, d}^{\prime}(x-d, y-d)\right), f\left(I_{\alpha, d}^{\prime}(x, y-d)\right), \\
& f\left(I_{\alpha, d}^{\prime}(x+d, y-d)\right), f\left(I_{\alpha, d}^{\prime}(x+d, y)\right), \\
& f\left(I_{\alpha, d}^{\prime}(x+d, y+d)\right), f\left(I_{\alpha, d}^{\prime}(x, y+d)\right), \\
& \left.f\left(I_{\alpha, d}^{\prime}(x-d, y+d)\right), f\left(I_{\alpha, d}^{\prime}(x-d, y)\right)\right\} .
\end{aligned}
$$

The directional descriptors with different $\alpha$ are concatenated to form the DCP descriptor as:

$$
D C P_{d}^{\prime}(x, y)=\left\{D C P_{\alpha_{i}, d}^{\prime}(x, y) \mid i=1,2,3,4\right\} .
$$

\begin{tabular}{|l|l|l|}
\hline$I_{X-1, y-1}$ & $I_{X, y-1}$ & $I_{X+1, y-1}$ \\
\hline$I_{X-1, y}$ & $I_{X, y}$ & $I_{X+1, y}$ \\
\hline$I_{X-1, y+1}$ & $I_{X, y+1}$ & $I_{X+1, y+1}$ \\
\hline
\end{tabular}

Fig. 1. An example of 8-neighborhood around $I_{x, y}$. 
Noted that the number of the direction $\alpha$ selected in different applications is diverse, considering that there is in practice a trade-off between computational efficiency and feature length.

\subsection{The similarity calculation based on Derivative Code and its pattern}

To exploit the proposed DerivativeCode method for palmprint recognition, we deploy the hamming distance on Derivative Codes. To the best of our knowledge, no people have used DerivativeCode for palmprint recognition before. The similarity of two DerivativeCodes $D c_{1}, D c_{2}$, can be calculated by using XOR operator:

$$
\operatorname{sim}=D c_{1} X O R D c_{2} .
$$

Different from the pixel-based DerivativeCode, DCP is proposed to capture the distribution information of the local pattern for faces, and we model DCP by spatial histogram, which can be considered as the region-based method. The method is similar to [10], but DCP is extension to our proposed DerivativeCode method that achieves the best performance on palmprint recognition. Furthermore, we do not include the DerivativeCode feature for current position in the DCP method, as we find it useless for the performance improvement, which is different from [10]. The image is spatially divided into non-overlapping rectangular regions (as shown in Fig. 2) represented by $R_{1}, \ldots, R_{L}$, from which spatial histograms $D C P$ are extracted as:

$$
H D C P(i)=\left\{H_{D C P}\left(R_{i}\right) \mid i=1, \ldots, L\right\},
$$

where $H_{D C P}\left(R_{i}\right)$ is the histogram of DCP extracted from the local region $R_{i}$. Noted that the regions do not need to be rectangular, and neither do they need to be of the same size or shape.

DCP is similar to LBP in some aspects, however, LBP just calculates the binary result between the center pixel and the surrounding ones, while DCP can extract the spatial information in a local region for a specified direction $[10,12]$.

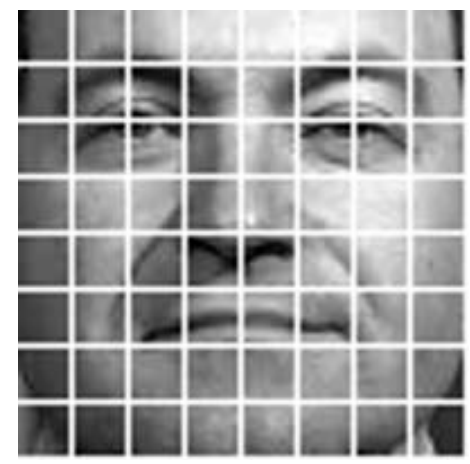

Fig. 2. Examples of a face divided into sub-regions. 
Many similarity measures for histogram matching have been proposed. In this paper, histogram intersection is used to calculate the similarity between two histograms:

$$
S_{H I}(\mathbf{H}, \mathbf{S})=\sum_{i=1}^{B} \min \left(H_{i}, S_{i}\right)
$$

where $S_{H I}(\mathbf{H}, \mathbf{S})$ is the histogram intersection statistic with $\mathbf{H}=\left(H_{1}, H_{2}, \ldots, H_{B}\right)^{T}$ and $\mathbf{S}=\left(S_{1}, S_{2}, \ldots, S_{B}\right)^{T}$. Moreover, similar to LBP and LDP that encode spatially varying patterns in local regions of an image $[9,15]$. Conceptually, it is anticipated that extending the proposed descriptor to feature images containing wider range of appropriate discriminative features could achieve a higher level of system performance. In image processing and object recognition, Gabor features are widely used image feature descriptors extracted by a set of Gabor wavelets (kernels) that model the receptive field profiles of cortical simple cells [9, 10, 30-33]. They can capture the salient visual properties in an image, such as spatial characteristics, because the kernels can selectively enhance features in certain scales and orientations. Here, we extend derivative code and its pattern to Gabor feature images to enhance the object representation capability. To calculate Gabor based on DerivativeCode, we use the Gabor filters as:

$$
\begin{aligned}
G(x, y, \theta, u, \sigma) & =\frac{1}{2 \pi \sigma^{2}} \exp \left\{-\frac{x^{2}+y^{2}}{2 \sigma^{2}}\right\} \\
& \exp \{2 \pi i(u x \cos \theta+u y \sin \theta)\},
\end{aligned}
$$

where $i=\sqrt{-1}$, $\mathrm{u}$ is the frequency of the sinusoidal wave, $\theta$ controls the orientation of the function and $\sigma$ is the standard deviation of the Gaussian envelope. Details about the parameters are shown in the experimental part.

\section{Experiments}

\subsection{Experiment on Palmprint Recognition}

We first do experiment on the PolyU palmprint database [13]. In this database, we have 600 images for 100 persons, with 6 images for each person. For DerivativeCode, we only use $0^{\circ}, 90^{\circ}$ to reduce the feature length. In the experiment on palmprint, we find the

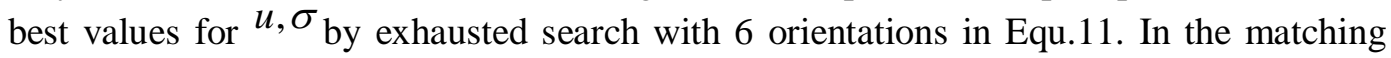
procedure, the maximum of the shift step is 4 in both horizontal and vertical directions. We demonstrate in Fig. 3 the distance distribution of genuine samples and imposters, and false acceptance rate in Fig. 4, which show that different people can be separated based on Derivativecode. It should be noted that the rotation is not considered as the palm print 
image is already normalized by two points, details about which can refer to [13]. Equal error rate (EER) of the state-of-the-art method (palmcode) in [8] is $0.34 \%$ on the PolyU database, but our method is 0 as shown in Fig. 4, which demonstrates that the DerivativeCode is more effective than palm code on the palm print identification.

To further investigate the performance of the proposed method, we also have tested it on a larger database with 7,752 images from 386 different palms [8]. Each of the palmprint images in the testing database is matched with all other palmprint images in the database to generate incorrect and correct identification hamming distances. EER of the method using the same parameter for Derivative Code is $0.03 \%$, but the best-reported result (palmcode) is $0.6 \%$ [8]. To further validate the performance of the proposed method, sparse representation classifier (SRC)[40] and sparse regression analysis (SRA) [5] are deployed for a comparison. Details about the methods are described in [5, 40]. As shown in Fig. 5, the proposed Derivativecode achieves a much better performance than SRA and SRC on the Polyu palmprint database. The number of training samples in SRA is one less than that in SRC in each class. In Fig. 5, '1-2' means that the images from 1st to 2nd are in the training set. ' $1-3$ ' and ' $1-4$ ' are similar to ' $1-2$ ' . It should be noted that all samples with the index 1 are included in the Gallery set for SRA;

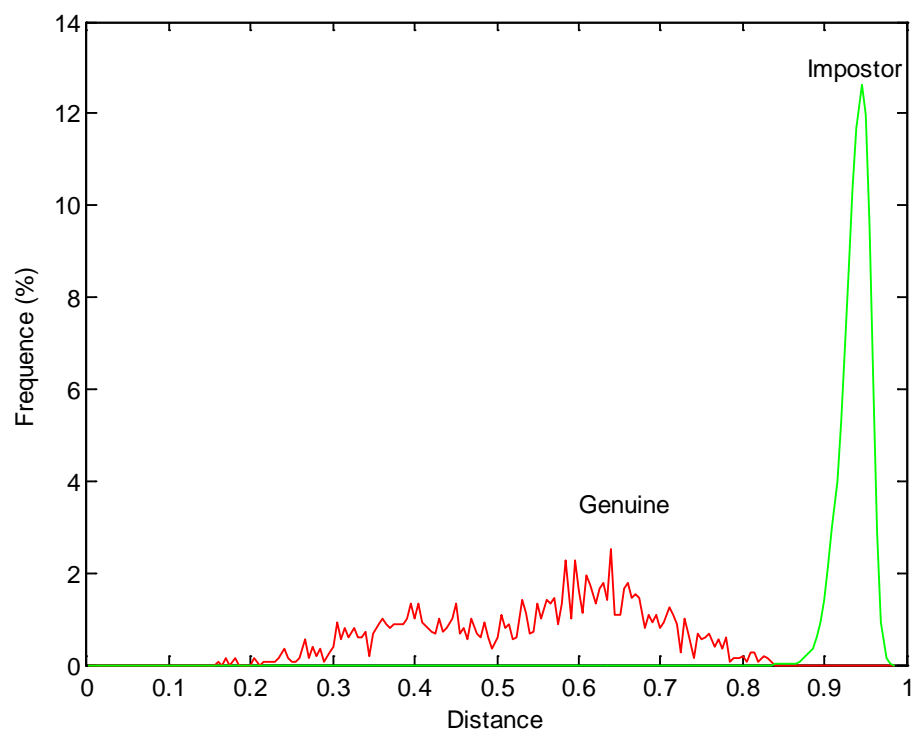

Fig. 3. The genuine and imposter distance distribution. 


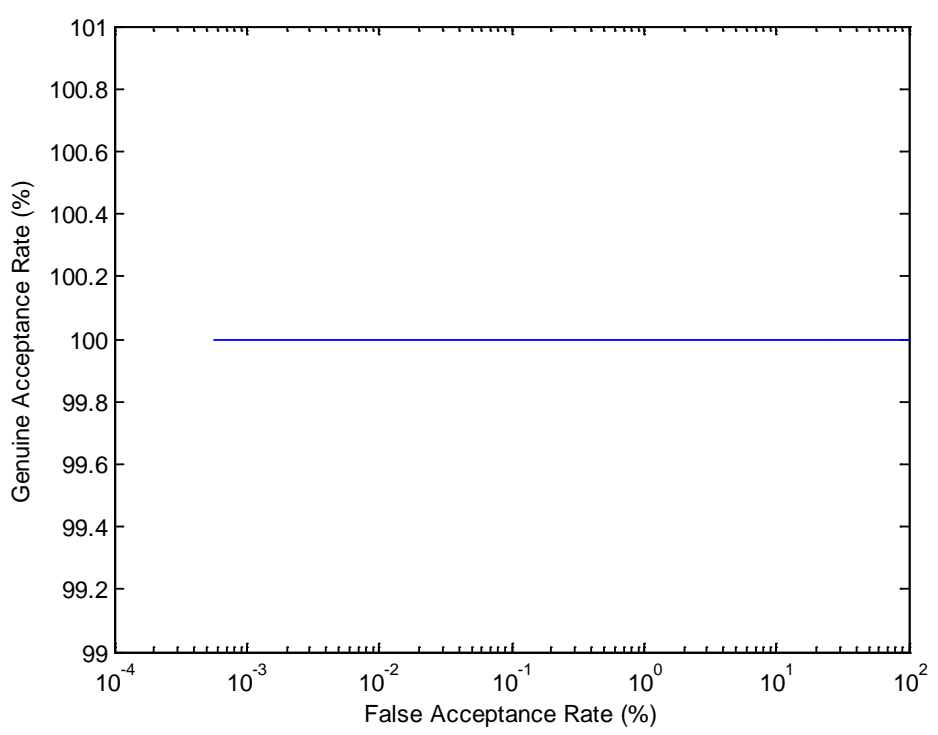

Fig. 4. The ROC curve of the PolyU database.

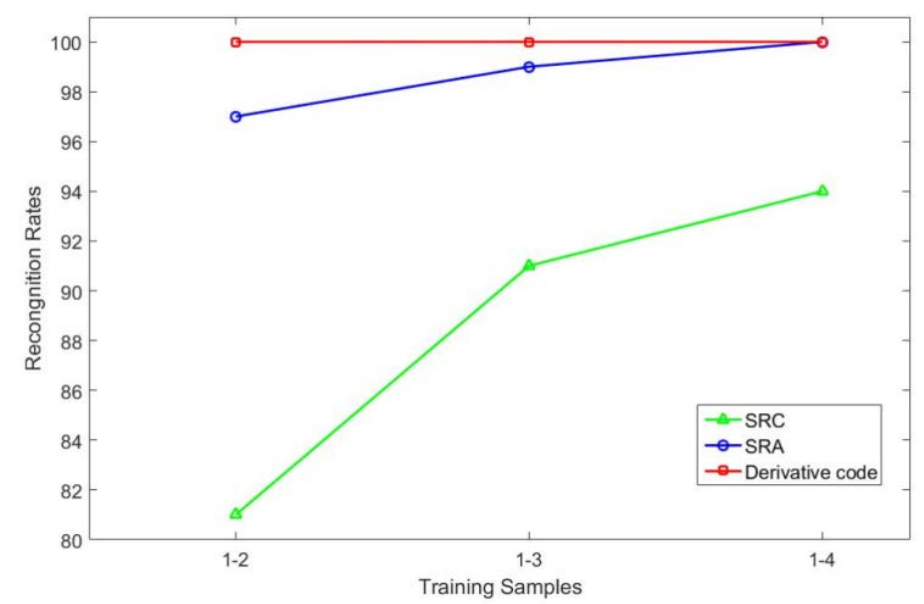

Fig. 5. The comparative results of Derivativecode, SRA and SRC on the Polyu palmprint database.

\subsection{Experiment on Face Recognition on the FERET database}

In this section, the comparative experiments between LBP and DCP are done on the FERET face database, which is widely exploited to evaluate face recognition algorithms [14]. The samples of FERET are shown in Fig. 8. In the experiments, the cropped face is normalized to $88 \times 88$ pixels. We use the same gallery and probe sets as in the standard FERET evaluation protocol. Fa containing 1196 frontal images of 1196 subjects is used as 
the gallery set, while Fb, Fc, Dup 1 and Dup 2 are used as the probe sets. We select the parameters of $11 \times 11$-sized sub-regions with 64 histogram bins for Gabor feature images. For DCP, d is chosen as 1 . Different from the experiments on palmprint databases, generally 5 frequency and 8 orientations are used in the face recognition [15]. And the parameters are as: $u=\pi, \pi / 2^{1 / 2}, \pi / 2, \pi / 2^{3 / 2}, \pi / 4$, with $\sigma=2 \pi$.

As shown in Fig. 6 and Fig. 7, the recognition accuracy of DCP is higher than that of LBP on the FERET dataset. For example, Gabor-DCP gets 7\% improvement than Gabor-LBP in the Dup 2 set. We believe that DCP can capture the spatial information in a local region for a specified orientation, while LBP just calculates the binary result between the center pixel and the neighbors. From experimental results as shown in Fig. 6 and Fig. 7, DCP is much better than LBP on Dup1 and Dup2, since DCP extracts more information than that of LBP and thus achieve a better performance when more variations in the datasets(Dup1 and Dup2). As shown in Table 1, the proposed method achieves a much better performance than some state-of-the results. We do not test SRC and SRA methods on the FERET database, since some subjects only have 1 sample in the Gallery set.

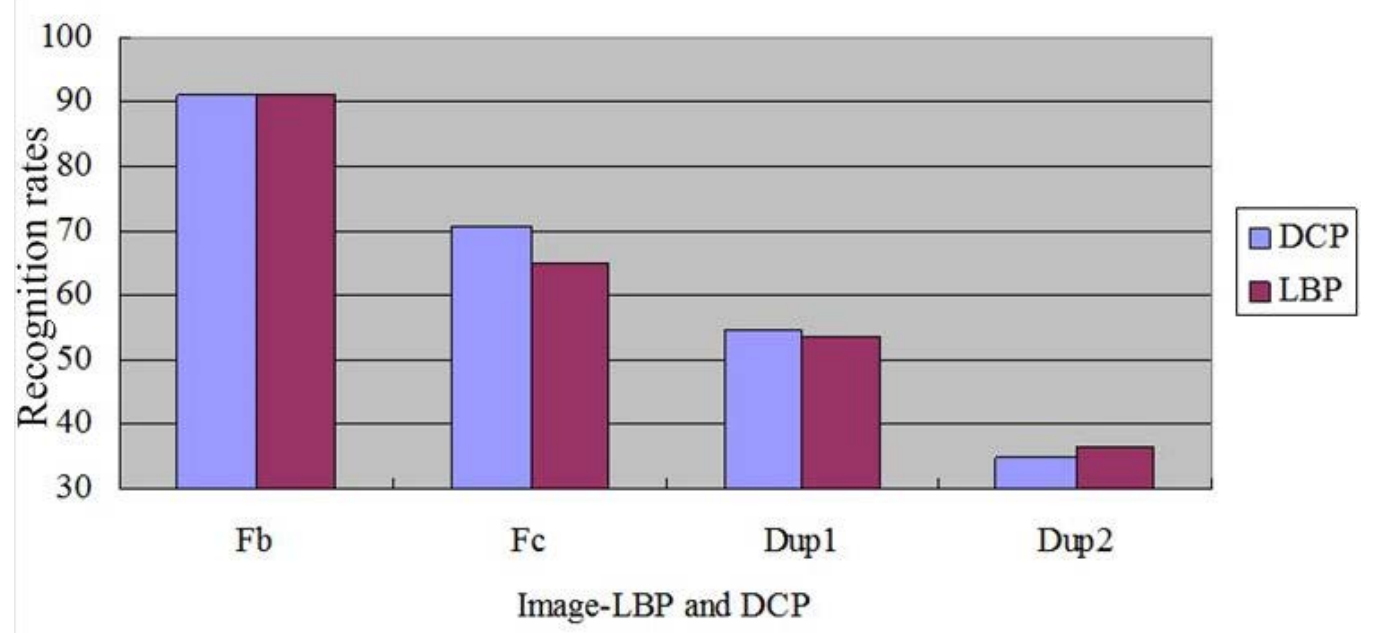

Fig. 6. Comparative results of image based LBP and DCP on FERET 


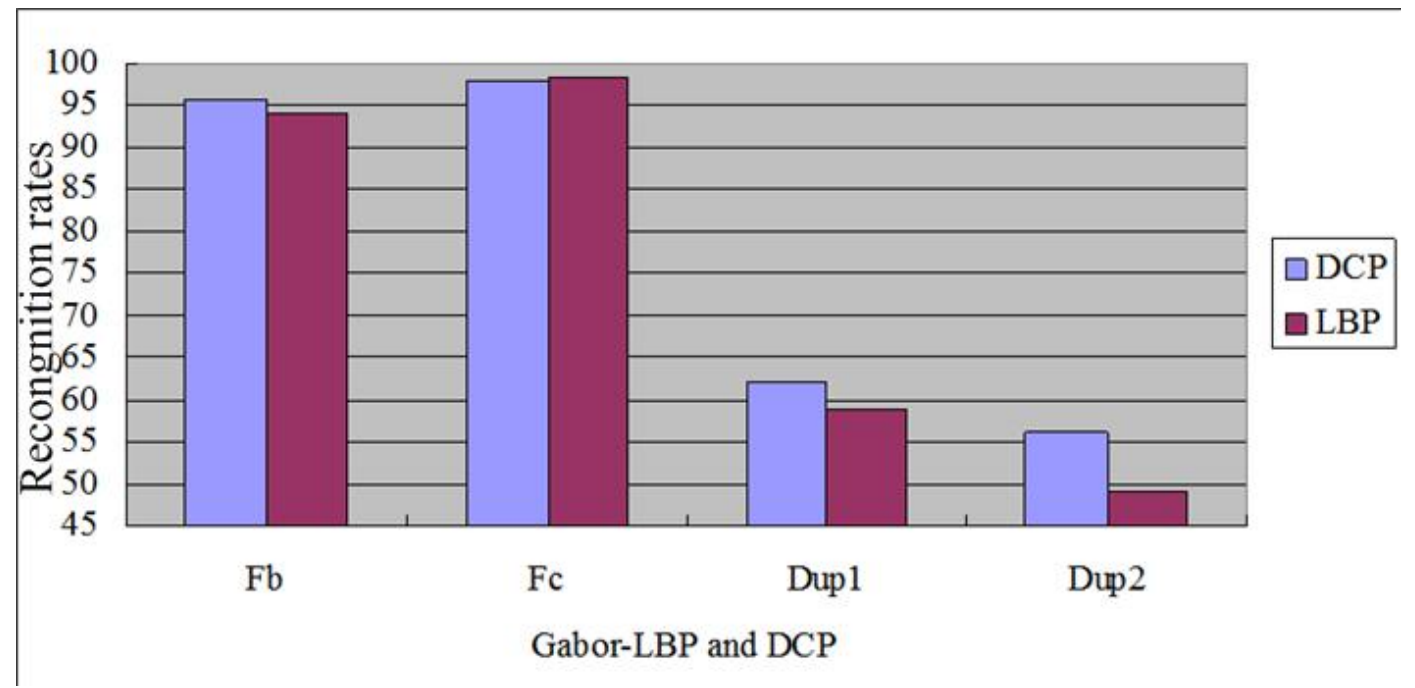

Fig. 7. Comparative results of Gabor based LBP and DCP on FERET

Table 1. Experimental results on the FERET database

\begin{tabular}{c|c|c|c|c}
\hline $\mathbf{\%}$ & Fisherface[18] & EBGM[18] & DCP & Gabor-DCP \\
\hline FaFb & 96.2 & 95.0 & 91.2 & 95.2 \\
\hline FC & 58.8 & 82.0 & 71 & 97.4 \\
\hline Dup1 & 47.2 & 59.1 & 52.1 & 62.2 \\
\hline Dup2 & 20.9 & 52.1 & 34.1 & 56 \\
\hline
\end{tabular}

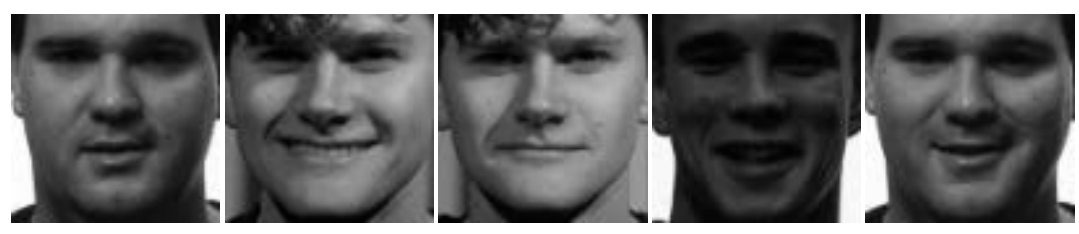

Fig. 8. The samples of the FERET database

\subsection{Experiment on the Polyu Infrared database}

We select two subsets from Polyu Infrared face database. In the Exp\#1, we use a subset from the whole PolyU-NIRFD database. In the subset, the training set contains 256 419 frontal images from 138 subjects, while the gallery set and probe set have 574 and 2,763 images 257 respectively. In the Exp\#2, we extracted from the whole database a much bigger subset than in the Exp\#1. In this subset, the training 295 set contains 1,876 frontal images of 150 subjects, while the gallery and probe sets have 1,159 and 4,747 
images, 296 respectively. No images in the query and target sets are contained in the training set. The facial portion of each original image is cropped based on the locations of the eyes. The cropped face is then normalized to $64 \times 64$ pixels. Eigenface, Fisherface, LBP methods are evaluated in the subsets, and their identification and verification performance are investigated. The distance in nearest neighbor classifier is the cosine of the angle between two representations in a PCA-space. For LBP histogram features, we use the histogram intersection similarity measure. We also compare the proposed method with SRC [40], sparse regression analysis (SRA) and kernelized sparse regression analysis (KSRA) [5].

As it can be seen from Table 2, using Gabor features of the original images has evidently improved the performance of Eigenface, Fisherface, LBP. The identification rate of Fisherface using Gabor features is $10 \%$ higher than that of the algorithms directly using grayscale images. It is interesting that the LBP method achieves a better performance than Eigenface and Fisherface. Both DCP and Gabor-DCP achieve much better performance than those of LBP and Gabor-LBP. Compared with SRC, SRA and KSRA, Gabor-DCP again achieves a much better performance, which further validate the performance of the proposed method.

Table 2. Experimental results on the Polyu infrared database

\begin{tabular}{c|c|c|c|c|c|c|c|c}
\hline $\mathbf{\%}$ & Fisherface[18] & LBP & Gabor-LBP & DCP & Gabor-DCP & SRC & SRA & KSRA \\
\hline Exp\#1 & 82 & 92.5 & 94.7 & 94 & 97 & 91.4 & 92.5 & 94.1 \\
\hline Exp\#2 & 86.2 & 84.3 & 89.3 & 86.7 & 92.1 & 89.1 & 90.2 & 92.4 \\
\hline
\end{tabular}

\section{Conclusion and Future Work}

This paper proposes a new method, Derivative Code (DerivativeCode) and its pattern for object recognition. The DerivativeCode is simply computed to capture the local relationship by using binary result of the mathematical derivative value. Gabor based DerivativeCode is successfully used on Palmprint recognition, which achieve much better performance than the state-of-the-art result on the PolyU database. Derivative code pattern (DCP) histogram is proposed to model local pattern feature for face recognition. Experimental results on the FERET face database and Polyu infrared face database show that the proposed DCP achieves better results than some state-of-the-arts.

Although the better performance we get on both palmprint and face recognition, the drawback of the proposed method lies in its feature length is a little big. To solve this problem, future work will focus on the statistic method to select the discriminative feature for an efficient object representation. Future work will apply our method on action recognition, tracking [27-30].

\section{Appendix:}

The pseudo code of the proposed method. Input: The input image I. 
Step1. The four derivatives at $\mathrm{I}(\mathrm{x}, \mathrm{y})$ is first calculated and further used to calculate the Derivativecode as:

$$
\begin{aligned}
& \operatorname{IOd}(x, y)=I(x, y)-I(x-d, y), \\
& \operatorname{I45d}(x, y)=I(x, y)-I(x-d, y-d), \\
& \operatorname{I90d}(x, y)=I(x, y)-I(x, y-d), \\
& \operatorname{I135d}(x, y)=I(x, y)-I(x+d, y-d) .
\end{aligned}
$$

Step2. The DerivativeCode is the pixel-based method to extract the directional feature, and derivative code pattern (DCP) derived from DerivativeCode in the direction $\alpha$ is accordingly calculated as:

$$
\begin{gathered}
\operatorname{DCP} \alpha d(x, y)=\{f(\operatorname{I\alpha d}(x-d, y-d)), f(\operatorname{I\alpha d}(x, y-d)), \\
f(\operatorname{I\alpha d}(x+d, y-d)), f((\operatorname{I\alpha d}(x+d, y)), \\
f(\operatorname{I\alpha d}(x+d, y+d)), f(\operatorname{I\alpha d}(x, y+d)), \\
f(\operatorname{I\alpha d}(x-d, y+d)), f(\operatorname{I\alpha d}(x-d, y))\} .
\end{gathered}
$$

Step3. The Gabor feature based Derivative Code descriptor

We first calculate Gabor features based on Equ. 11, and then Derivetivedcode and DCP are calculated using Step 1 and 2.

Step4. The similarity of two DerivativeCodes Dc1,Dc2 is calculated using the XOR operator as:

$$
\operatorname{sim}=D c 1 \text { XOR Dc2 }
$$

$$
\operatorname{HDCP}(i)=\{\operatorname{HDCP}(R i) \mid I=1, \ldots, L\}
$$

Similarly the similarity of two DCP patterns is calculated as follows:

$$
S_{H I}(\mathbf{H}, \mathbf{S})=\sum_{i=1}^{B} \min \left(H_{i}, S_{i}\right)
$$

OUTPUT: Recognition results.

\section{END}

\section{References}

[1] D. Marr, and E. Hildreth, "Theory of Edge Detection," in Proc. of the Royal Society of London, Series B, vol. 207, pp. 187-217, 1980. Article (CrossRef Link)

[2] W.T. Freeman, E. H. Adelson, "The Design and use of Steerable Filters," IEEE Transactions on Pattern Analysis and Machine Intelligence, vol.13, no.9, pp.891-906, 1991. Article (CrossRef Link)

[3] D. Gabor, "Theory of communication. Part 3: Frequency compression and expansion,” Journal of the Institution of Electrical Engineers-Part III: Radio and Communication Engineering, vol. 93, part III, no. 26, pp. 445-457, 1946. Article (CrossRef Link)

[4] X. Zhang and Y. Jia, "Local Steerable Phase(LSP) feature for face representation and recognition," in Proc. of IEEE International Conference on Computer Vision, pp.1363-1368, 2006. Article (CrossRef Link)

[5] Baochang Zhang, Suli Ji, Li Li, Shengping Zhang, and Wankou Yang, "Sparsity analysis versus sparse representation classifier,” Neurocomputing, vol.171, pp. 387-393, 2016.

Article (CrossRef Link) 
[6] Wangmeng Zuo, Zhouchen Lin, Zhenhua Guo, and David Zhang, "The Multiscale Competitive Code via Sparse Representation for Palmprint Verification," in Proc. of IEEE Conference on Computer Vision and Pattern Recognition, pp. 2265-2272, 2010. Article (CrossRef Link)

[7] T. Ahonen, A. Hadid, and M. Pietikäinen, "Face Description with Local Binary Patterns: Application to Face Recognition," IEEE Transactions on Pattern Analysis and Machine Intelligence, vol.28, no. 12, pp. 2037-2041, 2006.Article (CrossRef Link)

[8] David Zhang, Adams Wai-Kin Kong, Jane You, and Michael Wong, "Online Palmprint Identification," IEEE Transactions on Pattern Analysis and Machine Intelligence, vol. 25, no. 9, pp.1041-1050, 2003. Article (CrossRef Link)

[9] Baochang Zhang, Yongsheng Gao, Sanqiang Zhao, and Jianzhuang Liu, "Local Derivative Pattern Versus Local Binary Pattern: Face Recognition With High-Order Local Pattern Descriptor,” IEEE Transactions on Image Processing, vol.19, no.2, pp.533-544, 2010. Article (CrossRef Link)

[10] Baochang Zhang, Lei Zhang, David Zhang, and LinLin Shen, "Directional binary code with application to PolyU near-infrared face database,” Pattern Recognition Letters, vol. 31, no. 14, pp. 2337-2344, 2010. Article (CrossRef Link).

[11]Baochang Zhang, Alessandro Perina, Vittorio Murino, and Alessio Del Bue, "Sparse representation classification with manifold constraints transfer," in Proc. of CVPR, pp. 4557-4565, 2015. Article (CrossRef Link)

[12] Y. Gao and M.K.H. Leung, "Face Recognition Using Line Edge Map," IEEE Transactions on Pattern Analysis and Machine Intelligence, vol.24, no.6, pp.764-779, 2002. Article (CrossRef Link)

[13] PolyU Palmprint Palmprint Database, Article (CrossRef Link)

[14] P.J. Phillips, H. Moon, S.A. Rizvi, and P.J. Rauss, “The FERET Evaluation Methodology for Face-Recognition Algorithms," IEEE Transactions on Pattern Analysis and Machine Intelligence, vol. 22, no. 10, pp. 1090-1104, 2000. Article (CrossRef Link)

[15]B. Zhang, S. Shan, X. Chen, W. Gao, "Histogram of Gabor Phase Patterns: A novel object representation for face recognition,” IEEE Transaction on Image Processing, vol. 16, no. 1, pp. 57-58, 2007. Article (CrossRef Link)

[16]Zhihui Lai, Waikeung Wong, Yong Xu, Jian Yang, Jinhui Tang, and David Zhang, "Approximate orthogonal sparse embedding for dimensionality reduction," IEEE Transactions on Neural Networks and Learning Systems, vol.27, no.4, pp.723-735, 2016. Article (CrossRef Link)

[17] Shengping Zhang, Hongxun Yao, Huiyu Zhou, Xin Sun, and Shaohui Liu, "Robust Visual Tracking Based on Online Learning Sparse Representation,” Neurocomputing, Vol. 100, pp. 31-40, 2013. Article (CrossRef Link)

[18] P.J. Phillips, H. Moon, etc, "The FERET Evaluation Methodology for Face-Recognition Algorithms," IEEE Transactions on PAMI, vol. 22, no. 10, pp.1090-1104, 2000. Article (CrossRef Link)

[19] M.A. Turk, A.P. Pentland, "Face Recognition Using Eigenfaces," in Proc. of IEEE Conference on Computer Vision and Pattern Recognition, pp.586-5913-6, 1991 Article (CrossRef Link)

[20] M. Meytlis, L. Sirovich, "On the Dimensionality of Face Space," IEEE Transactions on Pattern Analysis and Machine Intelligence, Vol. 29, Issue 7, pp.1262 - 1267, 2007. Article (CrossRef Link)

[21] P. Belhumeur, J. Hespanha, and D. Kriegman, "Eigenfaces vs. Fisherfaces: Recognition using class specific linear projection," in Proc. of ECCV, pp.45-56, 1996. 
[22] A. M. martinez, Avinash C. Kak, "PCA versus LDA," IEEE Trans. Pattern Analysis and Machine Intelligence, vol. 23, no. 2, pp.228-233, 2001. Article (CrossRef Link)

[23]B. Schölkopf, A. Smola and K. Müller, "Nonlinear Component Analysis as a Kernel Eigenvalue Problem,” Neural Computation, vol.10, no.5, pp.1299-1319, 1998. Article (CrossRef Link)

[24] J. Yang, Alejandro F. Frangi, Jing-Yu Yang, David Zhang, and Zhong Jin, "KPCA Plus LDA: A Complete Kernel Fisher Discriminant Framework for Feature Extraction and Recognition," IEEE Transactions on Pattern Analysis and Machine Intelligence, vol. 27, no.2, pp. 230-244, 2005.Article (CrossRef Link)

[25] Penio S. Penev, and Joseph J. Atick, "Local Feature Analysis: a General Statistical Theory for Object Representation,” Network: Computation in Neural Systems, vol. 7, no. 3, pp. 477-500, 1996. Article (CrossRef Link)

[26] Y Cao, B Zhang, Z Guo, and J Liu, "Derivative code and its pattern for object recognition,” in Proc. of International Conference on Information \& Automation, vol. 43, no.10, pp.891-894, 2012. Article (CrossRef Link)

[27] Chen Chen, Mengyuan Liu, Baochang Zhang, Jungong Han, Junjun Jiang, and Hong Liu, "3D Action Recognition Using Multi-Temporal Depth Motion Maps and Fisher Vector," IJCAI, pp.3331-3337, 2016.

[28]Baochang Zhang, Alessandro Perina, Zhigang Li, Vittorio Murino, Jianzhuang Liu, and Rongrong Ji, "Bounding Multiple Gaussians Uncertainty with Application to Object Tracking," International Journal of Computer Vision, vol.118, no.3, pp. 364-379, 2016. Article (CrossRef Link).

[29] B. Zhang, Zhigang Li, Alessandro Perina, Alessio Del Bue, and Vittorio Murino, "Adaptive Local Movement Modelling (ALMM) for Object Tracking,” IEEE TCSVT, 2016. Article (CrossRef Link).

[30] Baochang Zhang, Alessandro Perina, Vittorio Murino, and Alessio Del Bue, "Sparse representation classification with manifold constraints transfer,” CVPR, pp.4557-4565, 2015. Article (CrossRef Link)

[31] Meng Yang, Pengfei Zhu, Feng Liu, and Linlin Shen, "Joint representation and pattern learning for robust face recognition,” neurocomputing, vol.168, no.30, pp. 70-80, 2015. Article (CrossRef Link)

[32] Zhihui Lai, Yong Xu, Qingcai Chen, Jian Yang, David Zhang, "Multilinear sparse principal component analysis," IEEE Transactions on Neural Networks and Learning Systems, vol. 25, no. 10, pp.1942-1950, 2014. Article (CrossRef Link)

[33] Xiaoshuang Shi, Zhenhua Guo, and Zhihui Lai, "Face recognition by sparse discriminant analysis via joint L2,1-norm minimization,” Pattern Recognition, vol.47,no.7,pp.2447-2453, 2014. Article (CrossRef Link)

[34] Zhihui Lai, Waikeung Wong, and Yong Xu, "Sparse alignment for robust tensor learning," IEEE Transactions on Neural Networks and Learning Systems, vol. 25, no.10, pp. 1779-1792, 2014. Article (CrossRef Link)

[35] Zexuan Zhu, Sen Jia, Shan He, Yiwen Sun, Zhen Ji, and Linlin Shen, "Three-dimensional gabor feature extraction for hyperspectral imagery classification using a memetic framework," Information Sciences, vol. 298, no. 1, pp.274-287,2015. Article (CrossRef Link)

[36] Linlin Shen, and Li Bai, "3D gabor wavelets for evaluating spm normalization algorithm," Medical Image Analysis, vol.12, no.3, pp.375-383, 2008. Article (CrossRef Link) 
[37] Linlin Shen, Li Bai, and Zhen Ji, "FPCODE: An efficient approach for multi-modal biometrics," International Journal of Pattern Recognition and Artificial Intelligence, vol.25, no.2, pp. 273-286, 2011.

[38] Yuwu Lu, Zhihui Lai, and Yong Xu, "Low rank preserving projections,” IEEE Transactions on Cybernetics, Article (CrossRef Link).

[39] Waikeung Wong, Zhihui Lai, Yong Xu and Jiajun Wen, "Joint tensor feature analysis for visual object recognition,” IEEE Transactions on Cybernetics, vol.45, no.11, pp.2425-2436, 2015. Article (CrossRef Link)

[40] J. Wright, A. Yang, A. Ganesh, S. Sastry and Y. Ma, "Robust Face Recognition via Sparse Representation," IEEE Transactions on Pattern Analysis and Machine Intelligence, vol.31. no.2, pp.210-227, 2009. Article (CrossRef Link)

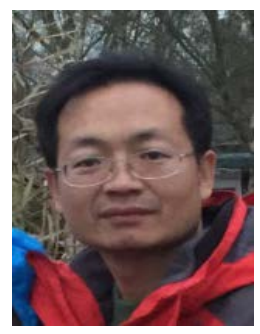

Baochang Zhang received the B.S., M.S., and Ph.D. degrees in computer science from the Harbin Institute of Technology, Harbin, China, in 1999, 2001, and 2006, respectively. From 2006 to 2008, he was a Research Fellow with the Chinese University of Hong Kong, Hong Kong, and with Griffith University, Brisbane, Australia. Currently, he is an associate professor with the Science and Technology on Aircraft Control Laboratory, School of Automation Science and Electrical Engineering, Beihang University, Beijing, China. He held a senior postdoc position in PAVIS department, IIT, Italy. He was supported by the Program for New Century Excellent Talents in University of Ministry of Education of China. His current research interests include pattern recognition, machine learning, face recognition, and wavelets.

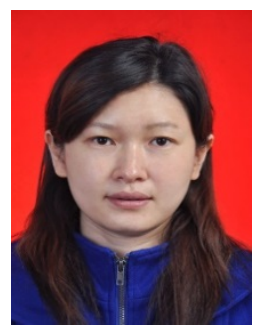

Hainan Wang is a Ph.D candidate in School of Automation Science and Electrical Engineering, Beihang university. She is also a lecturer with Guizhou university. Her research interests include pattern recognition and machine learning.

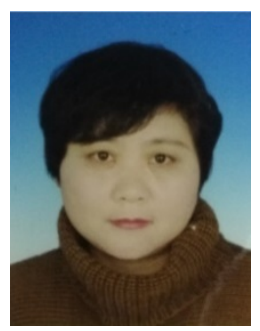

Hong Zheng received her Ph.D. degree in Harbin Institute of Technology, China, Currently, she is Professor with Beihang University. Her research interests include Hardware Design, Embedded System, pattern recognition, computer vision. 


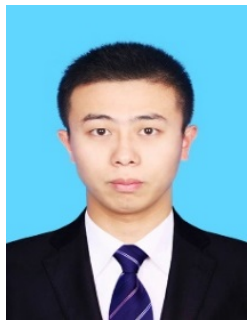

Yao cao received the B.Eng. and M.Eng. degrees from the Dalian Maritime University and the Beihang University, in 2010 and 2013, respectively. From 2013 to 2015, he was a Project Manager with the AVIC Avionics Co., Ltd., Beijing, China. From 2015 to 2016, he was a Senior Staff Member at the Bei-jing Municipal Commission of Housing and Urban-Rural Development. He is now a member of the Chi-nese Academy of Social Sciences Library, Beijing, China. His research interests include pattern recogni-tion, computer vision, machine learning, and information science.

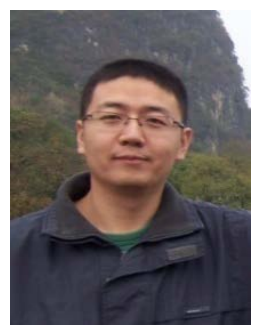

Zhenhua Guo received the M.S. and Ph.D degree in computer science from Harbin Institute of Technology and the Hong Kong Polytechnic University in 2004 and 2010 respectively. Since April 2010, he has been worked in Graduate School at Shenzhen, Tsinghua University. His research interests include pattern recognition, texture classification, biometrics, video surveillance, etc.

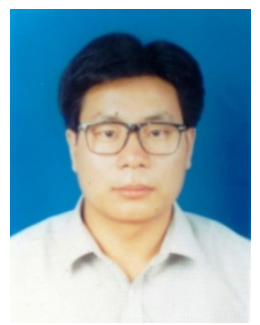

Chengshan Qian received the B.S., M.S., and Ph.D. degrees in electrical engineering, measuring and testing technologies and instruments, and control theory and control engineering from the Shandong University, Shandong University of Science and Technology, and Nanjing University of Aeronautics and Astronautics, China, in 1994, 2003, and 2009, respectively. Currently, he is a professor with the School of Computer \& Software, Nanjing University of Information Science \& Technology, Nanjing, China. His current research interests include intelligent control, nonlinear system control, and internet of things 